\title{
Numerical Investigation of Certain Asymptotic Results in the Theory of Partitions
}

\author{
By M. S. Cheema* and W. E. Conway
}

\begin{abstract}
A numerical investigation of some of the asymptotic formulas in partitions is made. Comparisons with actual computed values show that in certain cases only the relative error tends to zero and the errors are significant. Only in the case of $p_{k}(n)$ is it found that only a few terms of the asymptotic series are sufficient to obtain the exact value.
\end{abstract}

1. Introduction. Let $p_{k}(n)$ be defined by

$$
\prod_{i=1}^{\infty}\left(1-x^{i}\right)^{-k}=\sum_{n=0}^{\infty} p_{k}(n) x^{n}
$$

In an earlier paper, Cheema and Haskell [2] obtained asymptotic results for $p_{k}(n)$. These results are useful in obtaining similar results for $u_{k}(n)$, the number of $k$-line partitions of $n$. More recently, Gordon and Houten [5] derived the generating functions for $b_{k}(n)$, the number of $k$-line partitions of $n$, whose nonzero parts decrease strictly along rows, and extended their results to obtain the generating function for $b(n)$, the number of plane partitions of $n$, whose nonzero parts decrease strictly along rows. They also derived generating functions for the number of $k$-line and plane partitions under various other restrictions. MacMahon [7] had obtained the generating function for $u_{k}(n)$ and $u(n)$, the number of $k$-line and plane partitions of $n$, and Wright [12] obtained an asymptotic formula for $u(n)$. Following a similar technique, Gordon and Houten [4] have obtained an asymptotic formula for $b(n)$. In this article, we make a numerical investigation of some of these asymptotic results. The asymptotic results for $b(n)$ or $u(n)$, comparable in accuracy to those for $p(n)$ obtained by Hardy-Ramanujan-Rademacher, probably do not exist. Lehmer [8] investigated the asymptotic results for $p(n)$ and showed that $\alpha n^{1 / 2}$ terms of the series are sufficient so that the error will be less than $\frac{1}{2}$ in absolute value. The generating functions for $b(n)$ or $u(n)$ do not belong to the class of modular functions, thus making it impossible to obtain asymptotic formulas of the same type as that for $p(n)$. Our comparison with actual values of $b(n), b_{4}(n), p_{2}(n), p_{3}(n)$ show certain interesting features. Results indicate that the error terms in the asymptotic formulas for $b_{k}(n)$ and $b(n)$ are quite significant, and only the relative errors tend to zero as $n$ tends to infinity. Our computations confirm that results similar to those of Lehmer [8] also hold for the series for $p_{k}(n)$. Lehmer obtained estimates like $A_{q}(n)=O\left(q^{1 / 2+\epsilon}\right)$ for

Received August 16, 1971, revised January 11, 1972.

AMS 1970 subject classifications. Primary 10A45; Secondary 10A45.

Key words and phrases. Generating functions, $k$-line partitions, plane partitions, asymptotic formulas, modular functions, relative errors, Bernoulli numbers.

* Supported in part by NSF grant GP-12716.

Copyright (c) 1972, American Mathematical Society 
$A_{q}(n)$. Similar estimates have been obtained for $A_{q}(k, n)$ by Agarwal and Gandhi [1], with the extra condition that $(k, q)=1$. If one could extend these estimates for all $q$, then the proof that $\alpha n^{1 / 2}$ terms of the series for $p_{k}(n)$ are sufficient so that the error will be less than $\frac{1}{2}$ in absolute value will follow. Actual computations show the number of terms required is even much smaller.

2. Generating Functions. The generating functions for $p_{k}(n), u(n), u_{k}(n), b(n)$, and $b_{k}(n)$ are given by the following:

$$
\begin{aligned}
\prod_{i=1}^{\infty}\left(1-x^{i}\right)^{-k} & =\sum_{n=0}^{\infty} p_{k}(n) x^{n}, \\
\prod_{i=1}^{\infty}\left(1-x^{i}\right)^{-i} & =\sum_{n=0}^{\infty} u(n) x^{n}, \\
\prod_{i=1}^{\infty}\left(1-x^{i}\right)^{-\min (i, k)} & =\sum_{n=0}^{\infty} u_{k}(n) x^{n}, \\
\prod_{i=1}^{\infty}\left(1-x^{i}\right)^{-[(i+1) / 2]} & =\sum_{n=0}^{\infty} b(n) x^{n}, \\
P(x)^{[k / 2\}} Q(x)^{2\{k / 2]} \prod_{i=1}^{k-2}\left(1-x^{i}\right)^{[(k-i) / 2\}} & =\sum_{n=0}^{\infty} b_{k}(n) x^{n},
\end{aligned}
$$

where

$$
P(x)=\prod_{i=1}^{\infty}\left(1-x^{i}\right)^{-1}, \quad Q(x)=\prod_{i=1}^{\infty}\left(1-x^{2 i-1}\right)^{-1},
$$

$[k]$ and $\{k\}$ denote the integer and fractional part of $k$.

3. Asymptotic Results. These are stated by the following:

For $r \leqq 12$,

$$
p_{2 r}(n)=\frac{(r / 3)^{1 / 2}}{2^{r} \pi^{r-1} i} \sum_{q=1}^{\infty} A_{q}(2 r, n) q^{r-1} \frac{d^{r}}{d n^{r}}\left[\frac{J_{1}\left(\frac{2 \pi i\left(\frac{r}{3}\left(n-\frac{r}{12}\right)\right)^{1 / 2}}{q}\right)}{(n-r / 12)^{1 / 2}}\right] .
$$

For $2 r+1<24$,

$$
p_{2 r+1}(n)=\frac{1}{2^{r+1 / 2} \pi^{r+1}} \sum_{q=1}^{\infty} A_{q}(2 r+1, n) q^{r+1 / 2} \frac{d^{r+1}}{d n^{r+1}}
$$

$$
\cdot\left[\frac{\sinh \left(\frac{\pi\left(\frac{4 r+2}{3}\left(n-\frac{2 r+1}{24}\right)\right)^{1 / 2}}{q}\right)}{\left(n-\frac{2 r+1}{24}\right)^{1 / 2}}\right],
$$

where

$$
A_{q}(k, n)=\sum_{p}\left(\omega_{p, q}\right)^{k} \exp (-2 \pi i p n / q)
$$


$\omega_{p, \mathrm{~b}}$ being certain $24 q$ th roots of unity, summation extends over all $p, 1 \leqq p \leqq q$, such that $(p, q)=1$.

$$
b(n) \sim 2^{-3 / 4}(3 \pi \zeta(3))^{-1 / 2} N^{-49 / 24} \exp \left\{\frac{3}{2} \zeta(3) N^{2}+\frac{\pi^{2}}{24} N+C-\frac{\pi^{4}}{3456 \zeta(3)}\right\}
$$

where

$$
N=\left(\frac{n}{\zeta(3)}\right)^{1 / 3}, \quad C=\int_{0}^{\infty} \frac{y \log y}{e^{2 \pi y}-1} d y .
$$

A similar result for $u(n)$ is given in Wright [12]. Asymptotic results for $u_{k}(n)$ and $b_{k}(n)$ are derived from those of $p_{k}(n)$. In particular,

$$
\begin{gathered}
u_{k}(n) \sim(k-1) !(k-2) ! \cdots 2 ! \frac{d^{k(k-1) / 2}}{d n^{k(k-1) / 2}}\left(p_{k}(n)\right), \\
b_{4}(n) \sim \frac{1}{i \sqrt{ } 3} \frac{d^{3}}{d n^{3}}\left[\frac{J_{1}\left(2 \pi i\left(\frac{1}{3}\left(n-\frac{1}{12}\right)\right)\right)^{1 / 2}}{\left(n-\frac{1}{12}\right)^{1 / 2}}\right],
\end{gathered}
$$

which reduces to

$$
b_{4}(n) \sim \frac{\pi^{7}}{81} \sum_{m=0}^{\infty} \frac{\pi^{2 m}\left(\frac{1}{3}\left(n-\frac{1}{12}\right)\right)^{m}}{m !(m+4) !}
$$

Similarly, the asymptotic series for $p_{2}(n)$ and $p_{3}(n)$ reduce to

$$
\begin{gathered}
p_{2}(n)=\frac{1}{18} \sum_{q=1}^{\infty} A_{q}(2, n) \sum_{m=0}^{\infty} \frac{\pi^{2 m+3}\left(\frac{1}{3}\left(n-\frac{1}{12}\right)\right)^{m}}{m !(m+2) ! q^{2 m+3}}, \\
p_{3}(n)=\frac{1}{2^{3 / 2} \pi^{2}} \sum_{q=1}^{\infty} A_{q}(3, n) q^{3 / 2} \\
\cdot\left(\left(\frac{A-B}{2}\right) \exp \left(\frac{\pi}{q}\left(2 n-\frac{1}{4}\right)^{1 / 2}\right)-\left(\frac{A+B}{2}\right) \exp \left(-\frac{\pi}{q}\left(2 n-\frac{1}{4}\right)^{1 / 2}\right)\right)
\end{gathered}
$$

where

$$
A=\left(\frac{3}{2}+\frac{\pi^{2}}{q^{2}}\left(n-\frac{1}{8}\right)\right) / 2\left(n-\frac{1}{8}\right)^{5 / 2}, \quad B=\frac{3 \pi}{2 \sqrt{ } 2 q\left(n-\frac{1}{8}\right)^{2}} .
$$

The coefficients $A_{q}(n)=A_{q}(1, n)$ first occurred in Hardy-Ramanujan's asymptotic series for $p(n)$ and were studied by Lehmer [8], Whiteman [10], [11], Rademacher [9] and others. Selberg discovered the formula

$$
A_{q}(n)=\frac{1}{4}\left(\frac{k}{3}\right)^{1 / 2} \sum(-1)^{|l / 6|} \exp \left(\frac{\pi i l}{6 k}\right) .
$$

Summation is over integers $l$ such that $(l, 6)=1$ and $l^{2} \equiv 1-24 n(\bmod 24 q)$ and $|x|$ denotes the integer nearest to $x$. Such a formula should also exist for $A_{q}(k, n)$. Gandhi [3] has studied $A_{q}(k, n)$ and has obtained some of their properties. 
A table of $A_{q}(n)$ for $q \leqq 18$ was given by Hardy and Ramanujan [6]. It is easy to obtain a similar table for $A_{q}(k, n)$. For example,

$$
\begin{aligned}
& A_{1}(k, n)=1 \\
& A_{2}(k, n)=(-1)^{n}, \\
& A_{3}(k, n)=2 \cos \left(\frac{2 n \pi}{3}-\frac{k \pi}{18}\right), \\
& A_{4}(k, n)=2 \cos \left(\frac{n \pi}{2}-\frac{k \pi}{8}\right), \\
& A_{5}(k, n)=2 \cos \left(\frac{2 n \pi}{5}-\frac{k \pi}{5}\right)+2 \cos \left(\frac{4 n \pi}{5}\right) .
\end{aligned}
$$

For $k=1$, these reduce to the formulas obtained by Hardy and Ramanujan.

The value of $C$ in (3.3) was obtained by numerical integration of

$$
\int_{0}^{\infty} \frac{y \log (y)}{e^{2 \pi y}-1} d y=\int_{0}^{1} \frac{y \log y}{e^{2 \pi y}-1} d y+\int_{1}^{\infty} \frac{y \log (y)}{e^{2 \pi y}-1} d y .
$$

The second integral is easy to evaluate. The substitution $2 \pi y=u$ reduces the first integral to $\left(1 / 4 \pi^{2}\right) \int_{0}^{2 \pi}\left(u \log (u / 2 \pi) /\left(e^{u}-1\right)\right) d u$ which is written as

$$
\frac{1}{4 \pi^{2}} \int_{0}^{2 \pi}\left(\frac{u}{e^{u}-1}-1\right) \log \left(\frac{u}{2 \pi}\right) d u-\frac{1}{2 \pi}
$$

or as

$$
\frac{1}{4 \pi^{2}} \int_{0}^{\epsilon} \sum_{k=1}^{\infty} \frac{u^{k}}{k !} B_{k} \log \left(\frac{u}{2 \pi}\right) d u+\frac{1}{4 \pi^{2}} \int_{\epsilon}^{2 \pi}\left(\frac{u}{e^{u}-1}-1\right) \log \left(\frac{u}{2 \pi}\right) d u-\frac{1}{2 \pi}
$$

where $B_{k}$ are the well-known Bernoulli numbers. Taking $\epsilon=.1$ in these integrals and adding the second integral, we obtain $C=-.08271057185022965322$.

In the asymptotic calculations for $b(n), \zeta(3)=1.20205690315959428540$ and $\pi=3.14159265358979323846$ were used.

Numerical Results

$\mathrm{b}_{4}(\mathrm{n})$

\begin{tabular}{|r|r|r|}
\hline $\mathbf{n}$ & Exact Values & Asymptotic values \\
\hline 50 & 7861151 & 10769606 \\
\hline 100 & 81121690641 & 102674670000 \\
\hline 150 & 122723468142192 & 149502323956804 \\
\hline 200 & 65197255804176049 & 77556919390336123 \\
\hline 250 & 17410617101196225420 & 20368778539157277707 \\
\hline 299 & 2563183245773772069521 & 2961963804368820491881 \\
\hline
\end{tabular}


$b(n)$

\begin{tabular}{|r|r|r|}
\hline $\mathrm{n}$ & Exact Values & \multicolumn{1}{|c|}{ Asymptotic values } \\
\hline 50 & 134035641 & 131450402 \\
\hline 100 & 40271460800459 & 39515125499612 \\
\hline 150 & 1695070342263449292 & 1664761688894903178 \\
\hline 200 & 23318651621796353657014 & 22920072294220827550007 \\
\hline 250 & 150270591063287164944741750 & 147801858831116594095994117 \\
\hline 299 & 470334179683225117053159476103 & 462868009280107428736730772768 \\
\hline
\end{tabular}

4. Comments on Numerical Results. Comparisons of exact values with those given by asymptotic formulas for $b_{4}(n)$ and $b(n)$ indicate that the error in the asymptotic results are quite significant. However, in the case of $p_{2}(n)$ and $p_{3}(n)$, the asymptotic results are excellent. We were able to calculate the exact values of $p_{2}(50), p_{2}(100)$, $p_{3}(100)$, and $p_{3}(200)$ by the asymptotic formula. In fact, three terms of the asymptotic series gave the exact value of $p_{2}(50)$, while four terms were needed for $p_{2}(100)$. For $p_{3}(100)$, four terms of the series were needed, while for $p_{3}(200)$ it took six terms. The double precision accuracy of twenty eight digits for the CDC 6400 was exceeded by $p_{3}(299)$. However, the asymptotic series after four terms gave $p_{3}(299)=$ 37464893330090346069484200521 which is larger than the exact value by only 14 . This value was not improved by using additional terms up to and including eighteen, which is not surprising as we think the error is in the first four terms and is due to the limit on the accuracy of double precision arithmetic on the CDC 6400 .

The first six terms of the series for $p_{3}(200)$ are listed as are the first four terms of the series for $p_{2}(100)$, together with their exact values in the following table. In the appendix, we give the exact values of $b_{4}(n), b(n), p_{2}(n)$ and $p_{3}(n)$ for $n$ ranging from one to fifty only. The complete table up to two hundred and ninety nine has been published on the microfiche card that accompanies this issue of the journal. All computations were made on the CDC 6400 at the University of Arizona Computing Center.

First six terms of series for $p_{3}(200)$

56669336086583184772691.88617

875179402.76032

$-33461.76862$

64.90130

4.30801

.57670

sum $=56669336086584059918702.66388$

exact value $=56669336086584059918703$ 
First four terms of series for $p_{2}(100)$

$$
\begin{array}{r}
1843645804262.16325 \\
16512.32889 \\
-10.51372 \\
1.70633 \\
\text { sum }=1843645820765.68475 \\
\text { exact value }=1843645820766
\end{array}
$$

APPENDIX

n $b_{4}(n)$

b(n)

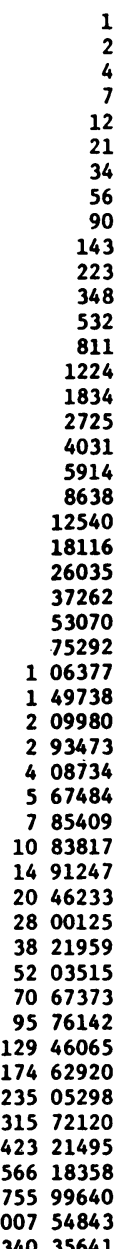

$p_{2}(n)$

$p_{3}(n)$

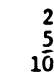

2
5
10

20
36

65

110

185

300

481

752

1165

1770

2665

3956

5822

8470

12230

17490

24842

35002

49010

68150

94235

129512

177087

240840

326015

439190

589128

786814

1046705

1386930

1831065

2408658

3157789

4126070

5374390

6978730

$90 \quad 35539$

11664896

15018300

19283830

24697480

31551450

40210481

51124970

64854575

82088400

103679156

$$
P_{3}(n)
$$

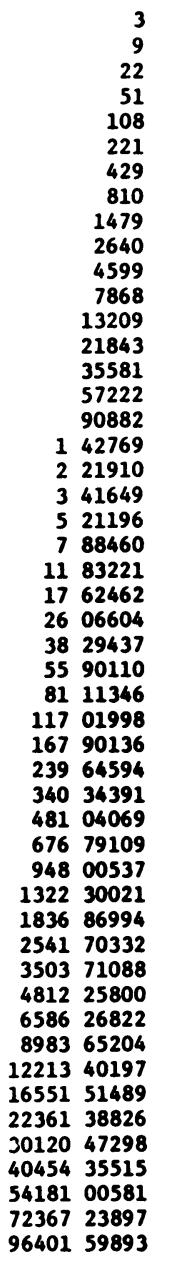

Department of Mathematics The University of Arizona Tucson, Arizona 85721 
1. S. K. Agarwal \& J. M. Gandhi, "Generalization of a certain function related to the partition function." Private communication.

2. M. S. Cheema \& C. T. Haskell, "Multirestricted and rowed partitions," Duke Math. J., v. 34, 1967, pp. 443-451. MR 36 \#125.

3. J. M. GANDHI, "Generalization of a certain function related to the partition function," Mathematica (Cluj), v. 11 (34), 1969, pp. 245-251. MR 41 \#5316.

4. B. Gordon \& L. Houten, "Notes on plane partitions. III," Duke Math. J., v. 36, 1969 , pp. 801-824. MR 40 \#1358.

5. B. Gordon \& L. Houten, "Notes on plane partitions," J. Combinatorial Theory, v. 4, 1968, pp. 72-99. MR 36 \#1339.

6. G. H. Hardy \& S. RamanuJan, "Asymptotic formulae in combinatorial analysis," Proc. London Math. Soc., v. 17, 1918, pp. 75-115.

7. P. A. MacMahon, Combinatory Analysis. Vols. I, II, Chelsea, New York, 1960. MR 25 \# 5003.

8. D. H. Lehmer, "The series for the partition function," Trans. Amer. Math. Soc., v. 43,1938 , pp. 271-295.

9. HANS RADEMACHeR, “On the Selberg formula for $A_{k}(n)$," J. Indian Math. Soc., v. 21, 1957, pp. 41-55. MR 19, 1163.

10. A. L. WhITEMAN, "A sum connected with the series for the partition function," Pacific J. Math., v. 6, 1956, pp. 159-176. MR 18, 195.

11. A. L. Whiteman, "A sum connected with the partition function," Bull. Amer. Math. Soc., v. 53, 1947, pp. 598-603. MR 8, 567.

12. E. M. WRIGHT, "Asymptotic partition formula. I. Plane partitions," Quart. J. Math., v. 2, 1931, pp. 177-189. 